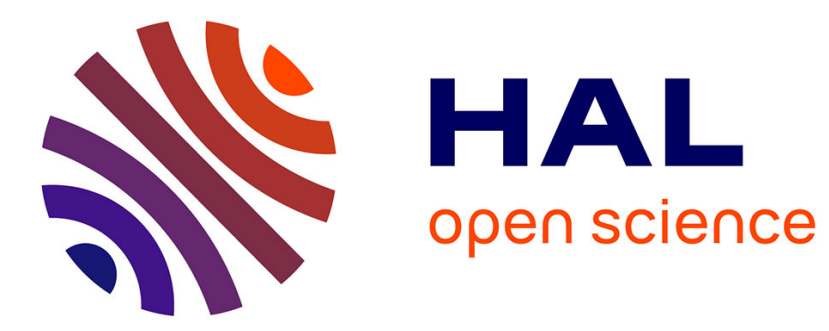

\title{
Prise en compte du mouvement dans la détermination numérique des courants de Foucault dans une structure électromagnétique
}

\author{
F. Bouillault, A. Abdel-Razek
}

\section{- To cite this version:}

F. Bouillault, A. Abdel-Razek. Prise en compte du mouvement dans la détermination numérique des courants de Foucault dans une structure électromagnétique. Revue de Physique Appliquée, 1983, 18 (2), pp.103-106. 10.1051/rphysap:01983001802010300 . jpa-00245069

\section{HAL Id: jpa-00245069 https://hal.science/jpa-00245069}

Submitted on 1 Jan 1983

HAL is a multi-disciplinary open access archive for the deposit and dissemination of scientific research documents, whether they are published or not. The documents may come from teaching and research institutions in France or abroad, or from public or private research centers.
L'archive ouverte pluridisciplinaire HAL, est destinée au dépôt et à la diffusion de documents scientifiques de niveau recherche, publiés ou non, émanant des établissements d'enseignement et de recherche français ou étrangers, des laboratoires publics ou privés. 
Classification

Physics Abstracts

$41.10 \mathrm{Fs}-02.70+\mathrm{d}-03.50 \mathrm{De}-89.20+\mathrm{a}$

\title{
Prise en compte du mouvement dans la détermination numérique des courants de Foucault dans une structure électromagnétique
}

\author{
F. Bouillault et A. Abdel-Razek \\ Laboratoire de Génie Electrique de Paris, E.S.E. $\left(^{*}\right)$, Univ. Paris VI et Paris XI, \\ Plateau du Moulon, 91190 Gif/Yvette, France
}

(Reçu le 8 juillet 1982, révisé le 8 novembre, accepté le 15 novembre 1982)

\begin{abstract}
Résumé. - La détermination des courants de Foucault dans les systèmes électromagnétiques par une méthode d'éléments finis nécessite souvent une modélisation dynamique. Les auteurs rendant compte du mouvement grâce à l'utilisation d'un macro-élément, déterminent l'expression discrétisée des courants en tous points du système dans un repère approprié. Ceci étant fait, ils exposent l'algorithme permettant de trouver les courants de Foucault et donnent un exemple de calcul.
\end{abstract}

\begin{abstract}
The determination of Eddy currents in electromagnetic systems, with the help of the finite element method, needs often the use of a dynamic model. To consider movement, the authors determine the expression of Eddy current, with the aid of a macro-element, using an appropriate reference system. They give the numerical algorithm to find these currents and give a numerical application.
\end{abstract}

1. Introduction. - L'optimisation des machines électriques nécessite la connaissance de la répartition spatio-temporelle du champ électromagnétique.

Il faut pour cela résoudre des équations de la forme :

$$
\boldsymbol{\nabla} \wedge(1 / \mu \boldsymbol{\nabla} \wedge \mathbf{A})=\mathbf{J}_{0}+\mathbf{J}_{\mathrm{f}}
$$

avec $\mathbf{A}$ potentiel vecteur,

$\mu$ perméabilité magnétique,

$\mathbf{J}_{0}$ courants de source,

$\mathbf{J}_{\mathrm{f}}$ courants induits.

Pour des structures compliquées, le calcul numérique peut être d'une grande utilité $[1,2]$. Il permet de déterminer les grandeurs physiques en différents points du système étudié.

Dans un problème bidimensionnel et linéaire; $\mathbf{A}=(0,0, A)$, l'équation (1) devient :

$$
\frac{\partial^{2} A}{\partial x^{2}}+\frac{\partial^{2} A}{\partial y^{2}}=-\mu\left(J_{0}+J_{\mathrm{f}}\right) .
$$

La méthode des éléments finis s'est avérée la plus précise avec un temps de calcul raisonnable. Dans cette méthode : les équations matricielles correspondant à (2) sont :

$$
[S][A]=\left[I_{0}\right]+\left[I_{\mathrm{f}}\right],
$$

(*) L.A. au CNRS nº 127.
[A] vecteur colonne des potentiels vecteurs aux nœuds du maillage,

$\left[I_{0}\right]$ intensités des courants de sources ramenées aux noeuds,

$\left[I_{f}\right]$ intensités des courants induits ramenées aux nouds,

$[S]$ matrice de raideur, tient compte de la position relative des différents milieux.

Si lors du mouvement la géométrie du système reste inchangée, on peut faire les calculs dans un repère fixe [3]. Si ce repère est choisi de telle façon que le mouvement se fasse suivant l'axe $x$, les courants de Foucault ont pour expression :

$$
J_{\mathrm{f}}=\sigma\left(-\frac{\partial A}{\partial t}-v_{x} \frac{\partial A}{\partial x}\right) .
$$

Cependant ceci conduit certes à une matrice $S$ invariante mais non symétrique [4], ce qui a pour effet entre autres d'augmenter l'espace mémoire nécessaire au calcul numérique. De plus, cette prise en compte du mouvement ne convient que pour des systèmes particuliers.

Dans le cas général, nous pouvons distinguer trois tentatives de prise en considération du mouvement : la topologie constante [5], la topologie variable et la topologie mixte [6,7]. Un examen de ces différentes méthodes permet de voir les inconvénients de ces 
méthodes [8]. L'utilisation d'un élément particulier "le macro-élément " permet de s'affranchir des difficultés de ces topologies. Le "macro-élément" est constitué d'un milieu linéaire dans lequel on sait résoudre analytiquement l'équation régissant le potentiel vecteur $[9,10]$. Le « macro-élément » va permettre la liaison entre les parties fixes du système et les parties mobiles.

2. Repère mobile. - Le choix du repère ayant une grande importance dans la technique de calcul utilisée, nous allons rappeler les formules de transformation des grandeurs physiques.

Le repère fixe sera un repère cartésien noté $R(x, y, z)$, le repère mobile noté $R^{\prime}\left(x^{\prime}, y^{\prime}, z^{\prime}\right)$ se déplace par rapport à $R$ à la vitesse $\mathbf{v} \equiv\left(v_{x}, 0,0\right)$ (Fig. 1).

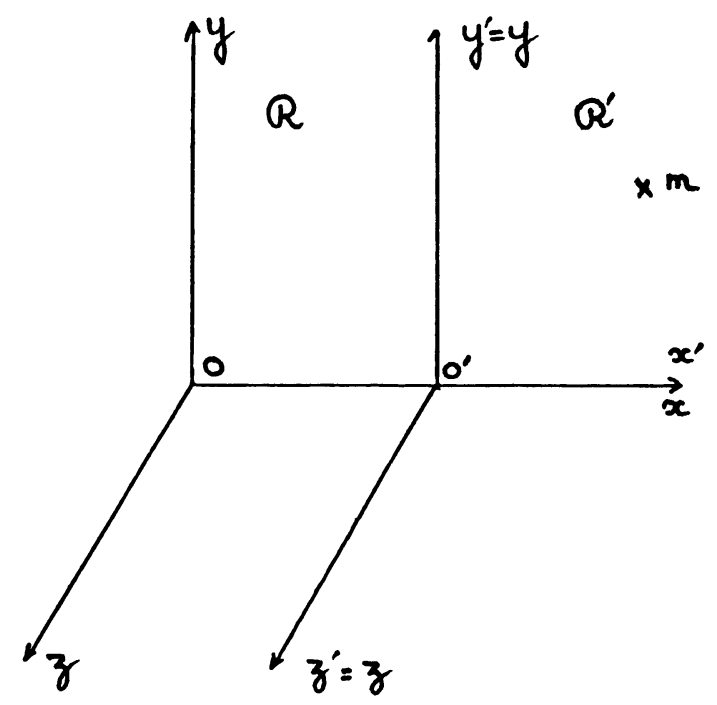

Fig. 1. - Repères $R$ et $R^{\prime}$.

[References $R$ and $R^{\prime}$.]

Si on modélise une machine, $R$ sera un repère lié au stator et $R^{\prime}$ lié au rotor. Toute grandeur relative à $R^{\prime}$ sera notée " '".

En électrotechnique, nous avons toujours $V x \ll C$ ( $C$ vitesse de la lumière) si bien que les correspondances sont les suivantes :

$$
\begin{aligned}
x^{\prime} & =x-v_{x} t \\
t^{\prime} & =t \\
\mathbf{A}^{\prime} & =\mathbf{A} \\
\mathbf{B}^{\prime} & =\mathbf{B} \\
\mathbf{E}^{\prime} & =\mathbf{E}+\mathbf{v} \wedge \mathbf{B} \\
\mathbf{J}^{\prime} & =\mathbf{J} .
\end{aligned}
$$

Dans le cas bidimensionnel, on peut choisir le repère tel que $\mathbf{A}$ et $\mathbf{J}$ est une composante :

$$
\begin{aligned}
A^{\prime}\left(x^{\prime}, y, t\right) & =A(x, y, t) \\
J^{\prime}\left(x^{\prime}, y, t\right) & =J(x, y, t) .
\end{aligned}
$$

3. Courants de Foucault. - L'existence des courants de Foucault est liée à la variation, en fonction du temps, du champ électrique induit $E_{\mathrm{i}}$ lié à la matière. Il nous faut considérer deux cas, suivant que le point appartient au repère fixe $R$ ou au repère mobile $R^{\prime}$.

Dans un point $(\mathrm{m}) d u$ repère $R^{\prime}$.- Dans les parties ferromagnétiques où nous calculons des courants de Foucault, il n'y a pas de champ électrique extérieur appliqué, on peut écrire :

$$
J_{\mathrm{m}}^{\prime}(t)=\sigma E_{\mathrm{i}}^{\prime}=\sigma E^{\prime}
$$

le champ électrique $E^{\prime}$ est lié à $A^{\prime}$ par la relation :

$$
E^{\prime}=-\frac{\partial A^{\prime}}{\partial t}
$$

(7), (8) et (9) donnent :

$$
J_{\mathrm{m}}(t)=-\sigma \frac{\partial A^{\prime}}{\partial t} .
$$

En calcul numérique, on est souvent amené à discrétiser l'opérateur $« \partial / \partial t »$, on peut écrire :

$$
J_{\mathrm{m}}(t)=-\sigma \frac{A^{\prime}\left(x^{\prime}, t\right)-A^{\prime}\left(x^{\prime}, t-\Delta t\right)}{\Delta t}
$$

à partir de (7) et (11), on obtient :

$J_{\mathrm{m}}(t)=-\sigma \frac{A(x(t), t)-A(x(t-\Delta t), t-\Delta t)}{\Delta t}$.

Dans un point $(\mathrm{m})$ du repère $R$. -

$$
J_{\mathrm{m}}(t)=\sigma E=-\sigma \frac{\partial A}{\partial t}
$$

la discrétisation conduit à

$$
J_{\mathrm{m}}(t)=-\sigma \frac{A(x, t)-A(x, t-\Delta t)}{\Delta t} .
$$

4. Modélisation par éléments finis. - Supposons qu'on ait déterminé la répartition du potentiel vecteur à l'instant $t-\Delta t$ pour le maillage de la figure $2 a$ où :

- le milieu 1 est un milieu immobile magnétique de conductivité $\sigma_{1}$,

- le milieu 2 est constitué d'un milieu linéaire de conductivité nulle,

- le milieu 3 est un milieu magnétique de conductivité $\sigma_{3}$, de plus, il se déplace à la vitesse $V_{x}$ par rapport au milieu 1 .

Le milieu 2 étant linéaire, il peut être constitué du macro-élément $[8,10]$ dont la matrice de raideur sera notée $[S E]_{t-\Delta t}$.

La matrice de raideur totale peut être considérée comme l'assemblage de 3 matrices de raideur élémen- 


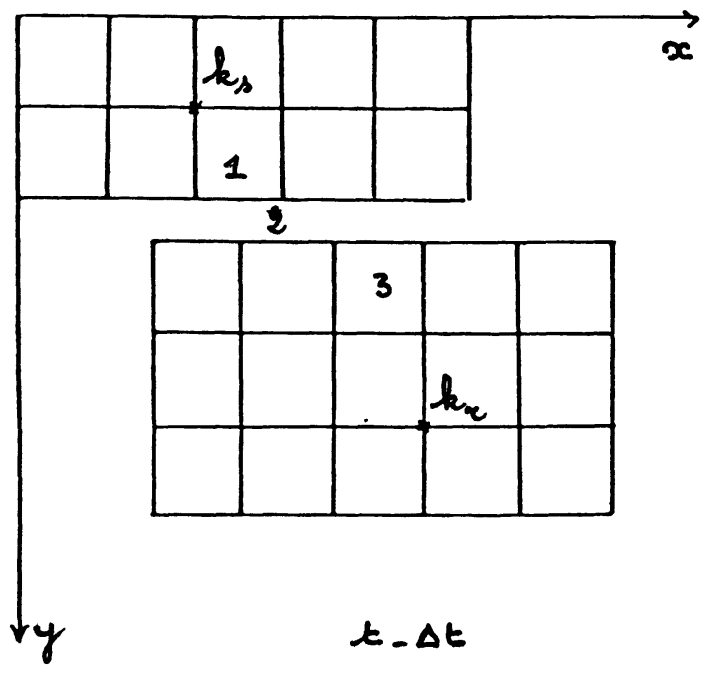

a)

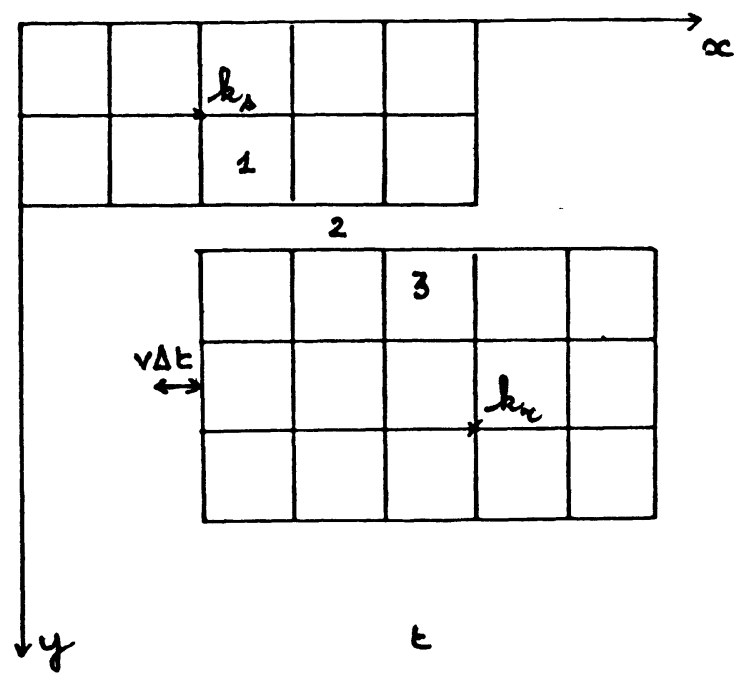

b)

Fig. 2. - Maillage aux instants $t-\Delta t$ et $t$.

[Mesh at the instants $t-\Delta t$ and $t$.]

taires $\left[S_{1}\right]_{t-\Delta t},[S E]_{t-\Delta t},\left[S_{3}\right]_{t-\Delta t}$. On désire déterminer pour le même système physique la répartition de $A$ à l'instant $\Delta t$ plus tard, pour cela, on prend le maillage de la figure $2 b$.

Pour calculer la matrice de raideur à l'instant $t$, il suffit de calculer $[S E]$, puisque $\left[S_{1}\right]$ et $\left[S_{3}\right]$ restent inchangées (le milieu 1 est fixe et le milieu 3 s'est déplacé en bloc de $v \Delta t$ ).

(2) devient à l'instant $t$

$$
[S]_{t}[A]_{t}=\left[I_{0}\right]_{t}+\left[I_{\mathrm{f}}\right]_{t}
$$

$\left[I_{\mathrm{f}}\right]_{t}$ peut se mettre sous la forme $[N]\left[J_{\mathrm{f}}\right]_{t}$ où :

- $[N]$ est la matrice de conduction résultant de l'assemblage de $\left[N_{1}\right]$ et $\left[N_{3}\right]$ (dans le milieu 2 il n'y a pas de courants de Foucault donc $\left[N_{2}\right]=[0]$ ),
- $\left[J_{\mathrm{f}}\right]_{t}$ vecteur colonne constitué des densités de courants induits aux nœuds de maillage.

Pour calculer les composantes $\left[J_{\mathrm{ft}}\right]$, la $k$-ième composante sera notée :

- $\mathrm{kr}$ si le point (m) appartient au milieu 3,

- ks si le point (m) appartient au milieu 1.

Si le point appartient au milieu 3 :

$J_{\mathrm{kr}}=-\sigma \frac{A\left(x_{\mathrm{kr}}(t), t\right)-A\left(x_{\mathrm{kr}}(t-\Delta t), t-\Delta t\right)}{\Delta t}$

les maillages aux instants $t$ et $t-\Delta t$ sont tels que

$$
\begin{gathered}
A\left(x_{\mathrm{kr}}(t), t\right) \equiv A_{\mathrm{kr}}(t) \\
A\left(x_{\mathrm{kr}}(t-\Delta t), t-\Delta t\right) \equiv A_{\mathrm{kr}}(t-\Delta t) \\
J_{\mathrm{kr}}=-\sigma \frac{A_{\mathrm{kr}}(t)-A_{\mathrm{kr}}(t-\Delta t)}{\Delta t}
\end{gathered}
$$

si le point appartient au milieu 1 :

$$
\begin{aligned}
J_{\mathrm{ks}} & =-\sigma \frac{A\left(x_{\mathrm{ks}}, t\right)-A\left(x_{\mathrm{ks}}, t-\Delta t\right)}{\Delta t} \\
& =-\sigma \frac{A_{\mathrm{ks}}(t)-A_{\mathrm{ks}}(t-\Delta t)}{\Delta t} .
\end{aligned}
$$

Donc quelle que soit la position du point :

$$
J_{\mathrm{k}}=-\sigma \frac{A_{\mathrm{k}}(t)-A_{\mathrm{k}}(t-\Delta t)}{\Delta t}
$$

ceci conduit à

$$
\left[I_{\mathrm{f}}\right]_{t}=-[N] \frac{[A]_{t}-[A]_{t-\Delta t}}{\Delta t}
$$

compte-tenu de l'expression (21), (15) devient :

$$
\left\{[S]_{t}+\frac{[N]}{\Delta t}\right\}[A]_{t}=\left[I_{0}\right]_{t}+\frac{[N]}{\Delta t}[A]_{t-\Delta t} .
$$

La résolution de l'algorithme (22) permet de déterminer le potentiel vecteur à tout instant et par la suite les courants de Foucault à partir de (21).

5. Résultats. - Nous avons modélisé une machine à rotor massif, alimentée par des bobinages statoriques triphasés. Les enroulements sont parcourus par des courants dont la densité est :

$$
J=J_{0} \cos 2 \omega_{0} t+\frac{1}{2} J_{0} \cos 4 \omega_{0} t .
$$

De plus, la machine est supposée tourner à la vitesse angulaire $\Omega=\omega_{0} / p$ où $p$ est le nombre de paire de pôles.

-Avec ces courants d'alimentation, le rotor voit deux champs tournant l'un à la vitesse de rotation $\Omega_{1}=$ $\omega_{0} / p$, l'autre à la vitesse $\Omega_{3}=3 \omega_{0} / p$. Ces résultats sont confirmés par la courbe de la figure 3 .

La courbe en pointillés est reconstituée à partir des harmoniques 1 et 3 de la courbe en traits pleins. 


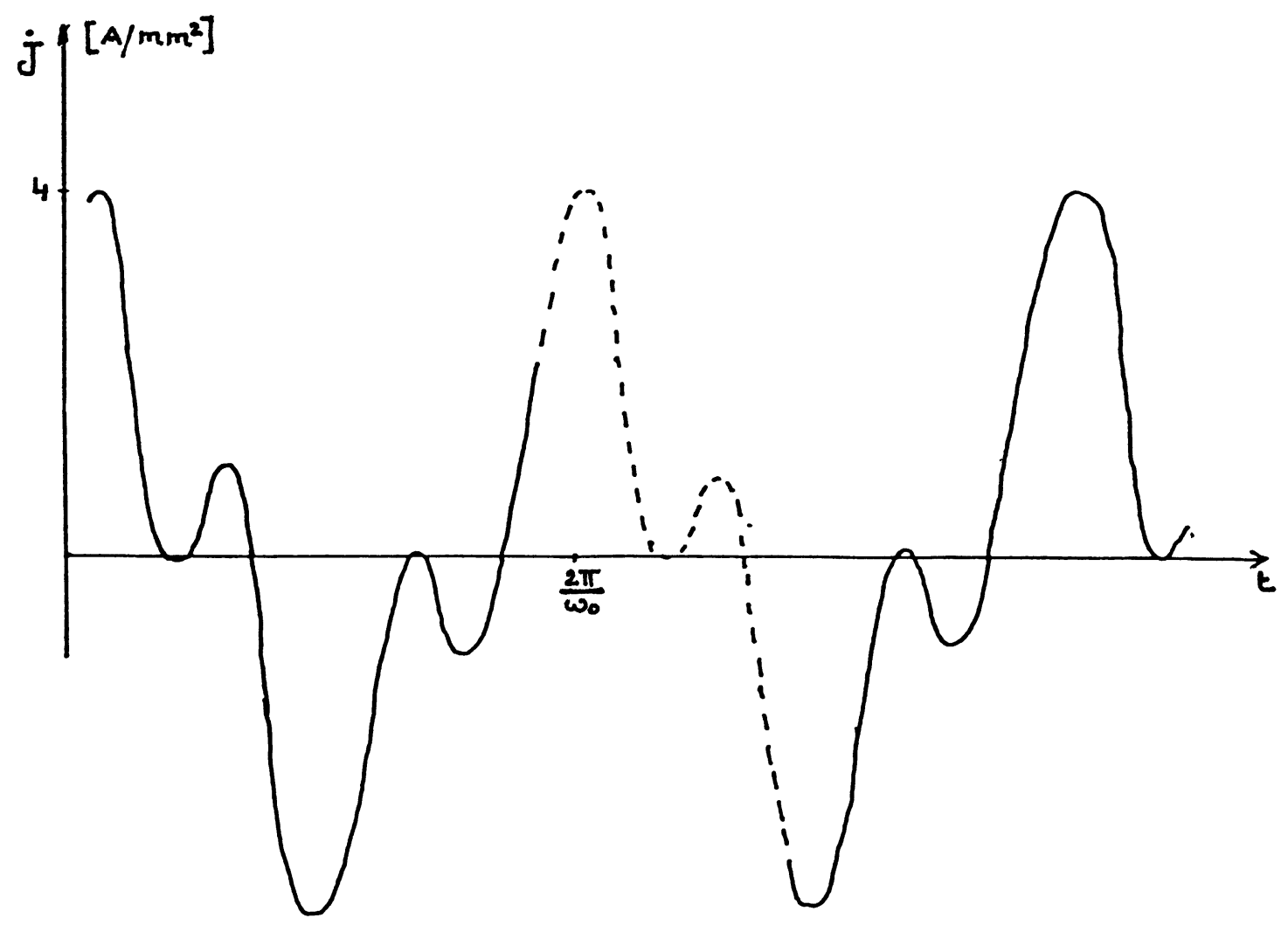

Fig. 3. - Courants de Foucault en un point rotor.

[Eddy currents at a point of the rotor surface.]

6. Conclusion. - La technique de calcul proposée permet de garder une matrice de raideur symétrique, ceci facilite les inversions de matrice et réduit l'espace mémoire nécessaire. De plus, on peut modéliser des systèmes dont la géométrie change avec le mouvement. L'utilisation du macro-élément permet de conserver la précision du calcul tout au long de la rotation et évite une renumérotation après quelques pas de calcul.

\section{Bibliographie}

[1] Silvester, P., Cabayan, H. S. and Browne, B. T., Efficient techniques for finite element analysis of electric machines. I.E.E.E. Trans. Power App. Syst. 92 (1973) 1274-1281.

[2] Jokl, A. L., Amstutz, L. J. and Erdelyi, E. A., Nonsymmetrical, nolinear aerospace alternators on non linear loads. I.E.E.E. Trans. Aerospace, Electron. Syst. vol. Aes (1975) 298-315.

[3] Foggia, A. et Sabonnadière, J. C., Les équations de Maxwell en électrotechnique et leur résolution numérique par une méthode d'éléments finis. Revue Phys. Appl. 14 (1979) 439-444.

[4] Foggia, A., Contribution à l'étude de l'induction magnétique dans les convertisseurs électromécaniques linéaires. Thèse de Docteur es Sciences Physiques, 1974.

[5] Feliachi, M., Contribution au calcul du champ électromagnétique par la méthode des éléments finis en vue d'une modélisation dynamique de machines électriques. Thèse de Docteur-Ingénieur, L.G.E.P., Paris, 1981.

[6] Glowinski, R. et Marrocco, A., Etude numérique du champ magnétique dans un alternateur tétra- polaire par la méthode des éléments finis, lecture Notes in Computer Science (Springer-Verlag) 10 (1973) 392-409.

[7] Berna, M., Contribution à l'étude d'un convertisseur électrique universalisé. Thèse de Docteur-Ingénieur, Paris, 1980.

[8] Felliachi, M. et Abdel-RazeK, A., Considération du mouvement du rotor de machines électriques dans une modélisation par éléments finis, Journée S.E.E. (Méthodes de calcul numérique en électrotechnique, Application aux machines électriques) Gif-sur-Yvette, 1981.

[9] Abdel-RazeK, A., Coulomb, J. L., Feliachi, M. and Sabonnadière, J. C., Conception of an Air Gap Element for dynamic Analysis of Electromagnetic fields in Electrical Machines. I.E.E.E. Trans. Mag. 18 (1982) 655-659.

[10] Felliachi, M., Abdel-Razek, A. and Boulllault, F., A synchronous machine dynamic model for magnetic field calculation. I.C.E.M., Budapest, 1982. 